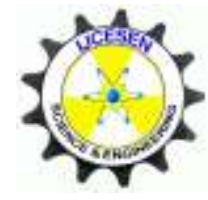

Copyright (C) IJCESEN
International Journal of Computational and

Experimental Science and $\boldsymbol{E N g i n e e r i n g}$

(IJCESEN)

Vol. 7-No.3 (2021) pp. 110-113

http://dergipark.org.tr/en/pub/ijcesen

Research Article

\title{
Modeling of the Magnetic Field of Current Carrying Conductor with Finite Elements Method
}

\author{
Naim SYLA ${ }^{1}$, Hajrudin HUSEJNI ${ }^{2 *}$, Gazmend NAFEZI ${ }^{3}$ and Fisnik ALIAJ ${ }^{4}$ \\ ${ }^{1}$ University of Prishtina, FMNS/Department of Physics, 10000 Prishtina, Kosovo \\ naim.syla@uni-pr.edu ORCID: 0000-0003-0857-4685 \\ ${ }^{2}$ University of Prishtina, FMNS/Department of Physics, 10000 Prishtina, Kosovo \\ * Corresponding Author : hajrudin@yahoo.com ORCID: 0000-0003-4100-5564 \\ ${ }^{3}$ University of Prishtina, FMNS/Department of Physics, 10000 Prishtina, Kosovo \\ gazmend.nafezi@uni-pr.edu ORCID: 0000-0002-9259-7259 \\ ${ }^{4}$ University of Prishtina, FMNS/Department of Physics, 10000 Prishtina, Kosovo \\ fisnik.aliaj@uni-pr.edu ORCID: 0000-0002-9967-8334
}

\section{Article Info:}

DOI: $10.22399 /$ ijcesen. 820667

Received: 03 November 2020

Accepted: 04 August 2021

\section{Keywords}

Magnetic field,

Conductor,

Modeling,

Finite Element Method,

Ansoft Maxwell

\begin{abstract}
:
In this paper, we have modeled and simulated the magnetic field of the conductor by using the finite element method, which is incorporated in Ansoft Maxwell software. Modeling and simulation are done for a conductor and two conductors located at a small distance between them. Our study is based on the method of finite elements and Biot-Savart law for calculating the magnetic field depending on the distance from the conductor. We have observed that the calculated values of the magnetic field are in good accordance with Biot-Savart law [1]. We have found that magnetic field normal to the main axis of the conductor falls linearly towards it, whereas outside the conductor magnetic field is inversely proportional to distance.

In the case of simultaneous calculation of the field inside and outside the conductor, a small margin has been found. This difference is as a result of the simultaneous calculation of magnetic field in two environments with different magnetic permeability.

Finally, we have computed the magnetic field at an equal point between two conductors when the currents have opposite and same direction, whereby a huge difference between the magnetic fields is found. We show that we can amplify or reduce the intensity of the magnetic field, based not only on the distance between the conductors but also on the basis of the directions of the currents flowing through these conductors.
\end{abstract}

\section{Introduction}

The magnetic field created by the current-carrying conductor can be calculated by Biot-Savart law. The magnetic field at a given point depends on several factors such as the length of the conductor, the intensity, the distance of the point from the conductor and the angle that the current element closes with the point where the magnetic field is required. On the other hand, in the case of two conductors, the magnetic field either can be amplified or weakened, depending on the distance between the two conductors and on the directions of currents flowing through these conductors. As a result, conductors either can attract or repel from each other. Magnetic fields are treated through Maxwell equations [2], which in most cases are difficult to be solved considering the complex nature of the problem (boundary conditions of integration). Therefore, approximate numerical methods should 
be applied in their solution. One of these methods is the Finite Elements Method (FEM). Characteristic of this method is the division of the research area (integration) into small suitable elements. Then all the necessary steps that lead to the solution of a certain problem are followed. In our case, Ansoft Maxwell software is used to model the magnetic field. In order for a problem to be solved through Ansoft (Ansys) Maxwell, we have to go through three stages: pre-processing, solving and postprocessing [3].

\section{Modeling and Simulation}

Modelling is the process of building the model, which must be compatible with the real system that has to be analyzed; while simulation is the process performed on the model, we have created using suitable software [3-11]. The advantage of modelling and simulation is the short time needed to solve any problem, low cost, testing of different situations, testing with different materials, etc. The most important steps for magnetic field modelling and analysis that we have followed are: defining the type of analysis, defining the type of element, constructing the (model) geometry, determining the properties of the material, applying boundary conditions and loads, defining the mesh, specifying of analysis for solutions as well as control (verification) of the results. Once the model is created, the automated Maxwell solution sequence takes over and fully controls the solution process without any interaction from the user.

We have dealt with three separate cases. In all three cases the dimensions of the conductor, in cylindrical shape, are the same. The conductor has a radius of $0.25 \mathrm{~mm}$ and length of $5 \mathrm{~mm}$. The conductor/s in the direction of $\mathrm{z}$ component are traversed by steady current with magnitude of $20 \mathrm{~A}$, while as a material for those conductors we have used copper due to its very good conductivity.

When modelling the magnetic field of currentcarrying conductor we not only should be careful in modelling correctly the geometry of the conductor, but also, we should be careful in the creation of region we wish to analyze magnetic field. If we want to determine the magnetic field inside the conductor, then the result is not affected by the size of the region, but on the other hand if we want to determine the magnetic field outside the conductor then the size of the region should be as large as possible, so as not to limit the convergence of (the lines of) magnetic field. In our case, we have given $300 \%$ padding in $x$ and $\mathrm{y}$ directions, whereas $0 \%$ padding in $\mathrm{z}$ direction
[3]. This is so because the magnetic field is normal to the $\mathrm{z}$ direction, figure 1.a. Regarding the mesh we have used an automatic adaptive mesh refinement technique, figure $1 . b$, while for the boundary condition we have selected default boundary

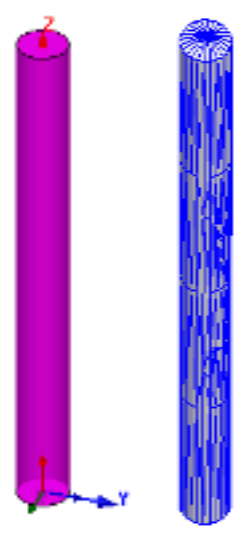

Figure1. Selected model and mesh

\section{Results}

In the following we will present three cases of magnetic field modelling: for a single conductor, and two parallel conductors where in one case the directions of currents flowing through conductors are parallel, while in the other case anti-parallel.

\subsection{The case of a single conductor}

After imposing boundary condition, Ansoft software automatically calculates the magnetic field of the conductor, figure 2 . While the change of magnetic induction intensity from the center of the conductor to an arbitrary point outside the conductor it will be as in figure 3 .
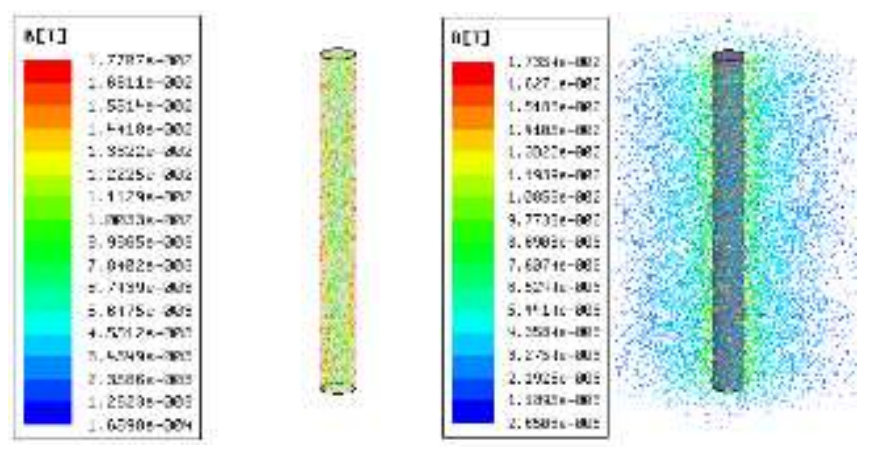

Figure 2. Value and distribution of magnetic induction inside and outside the conductor

\subsection{The case of two conductors in which parallel currents flow}

Same as for the conductor above the same parameters and material are used. Assume that these two conductors are located close to each other at a distance of $0.50 \mathrm{~mm}$. In the case of two conductors, 
the magnetic fields created by these current-carrying conductors interact with each other. The resulting magnetic field depends not only on the distance, the intensity of the currents, but also on the direction of the currents.
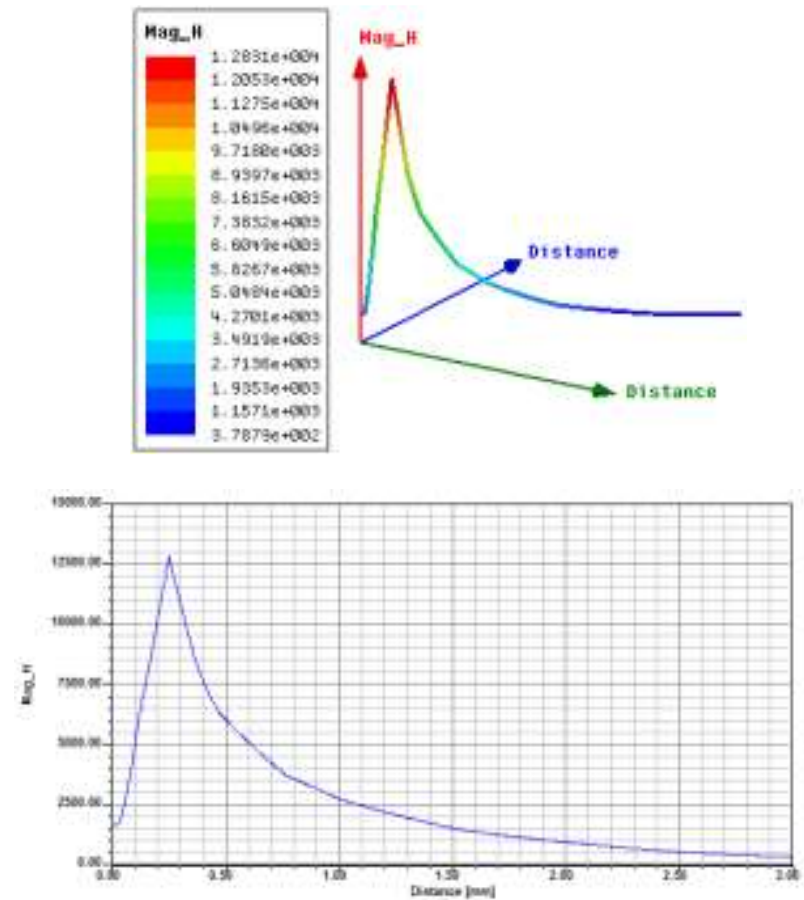

Figure 3. Intensity of the magnetic field of the conductor depending on the distance

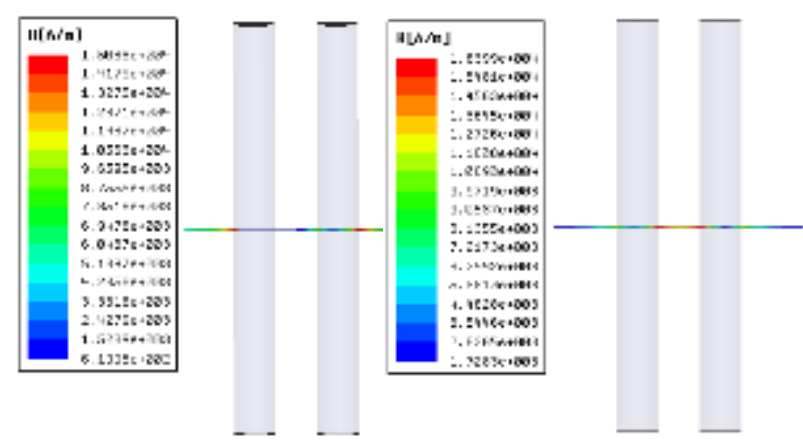

Figure 4. Magnetic field of two conductors for the cases when their currents are: $a$. parallel respectively $b$. antiparallel.

In the figure below we have presented the resulting magnetic field in cases where currents have the same direction and opposite direction, figure 4. For this case the dependence of the magnetic induction on the distance changes as is shown in Figure 5. As a result of the interaction of magnetic fields at the distance of $1.50 \mathrm{~mm}$ from the coordinate system, i.e. at the distance of $0.25 \mathrm{~mm}$ between the conductors, we have a significant decrease of the magnetic field.

\subsection{The case of two conductors through which} anti-parallel currents flow

All physical parameters are also preserved in this case, except that the currents flowing through the conductors once have the same direction and the next time have the opposite direction. Compared to the previous case, we not only have a noticeable amplification of the magnetic field in the distance between the two conductors, but we also have amplification in a certain part of the surface of the conductors. The dependence of the magnetic induction intensity on the distance in the occasion when currents are anti-parallel is shown in figure 6.
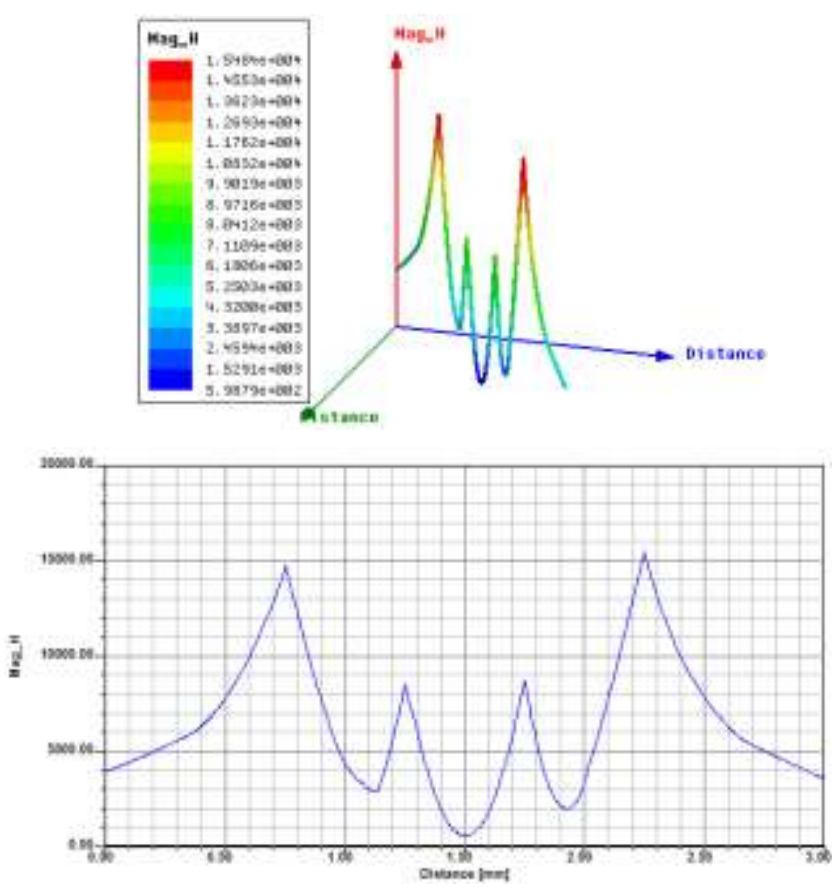

Figure 5.The change of magnetic field intensity depending on the distance when currents have the same directions

\section{Conclusions}

- Through modeling and simulation, we have the ability to visually represent the magnetic field of a current-carrying conductor and predict the results for even more complex cases.

- In the case of one conductor, we have found how the intensity of the magnetic field $(\mathrm{H})$ changes from inside to outside of the conductor. The magnetic field inside the conductor changes linearly with the conductor radius $\mathrm{B}=(\mu \mathrm{Ir}) /$ $\left(2 \pi R^{2}\right)$, while outside the conductor the magnetic field follows the law of Biot-Savart $\mathrm{B}=$ $\left(\mu_{0} I\right) /(2 \pi r)$ so as field falls off as $1 / r$, figure 3 . 

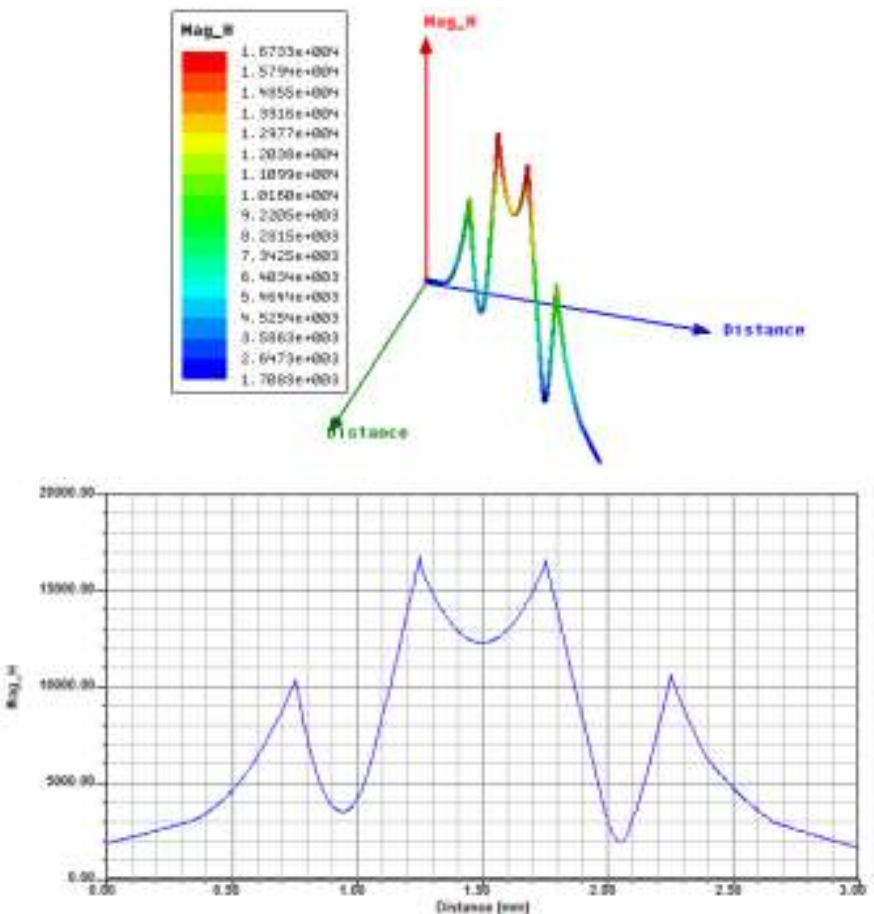

Figure 6. The change of magnetic field intensity depending on the distance between two conductors when currents have opposite directions.

- The magnetic field has minimum value near the main axis of the conductor, $\mathrm{H}=1710 \mathrm{~A} / \mathrm{m}$. The intensity of the magnetic field decreases significantly going towards the center due to the fact that the magnetic fields formed by each particle cancel each other out, so as we have weakening of the field, figure 3 .

- In the case of two conductors two situations arise: the amplification and the weakness of the magnetic field depending on the directions of the currents within the conductors.

- When currents have same direction, we notice that magnetic field between the conductors weakens. Exactly at the distance $0.25 \mathrm{~mm}$ between the conductors we have the minimum value $\mathrm{H}=598.78 \mathrm{~A} / \mathrm{m}$, while the magnetic field depending on the distance, changes as in figure 5

- While in the case when currents have opposite directions, we have a significant amplification of the magnetic field. Exactly in the middle of the distance between the conductors (i.e. d / $2=$ $0.25 \mathrm{~mm}$ ), the magnetic field has the value $\mathrm{H}=$ $12301 \mathrm{~A} / \mathrm{m}$. This amplification occurs because the magnetic field lines of the two conductors, in the space between them, have the same direction, so they do not cancel each other out, figure 6 . In this case, two maxima that belong to two separate parts of the surfaces of these conductors are clearly seen.

\section{Author Statements:}

- The authors declare that they have equal right on this paper.

- The authors declare that they have no known competing financial interests or personal relationships that could have appeared to influence the work reported in this paper

- The authors declare that they have nobody or nocompany to acknowledge.

\section{References}

[1] Mário H Oliveira and José A Miranda, Biot-Savartlike law in electrostatics, European Journal of Physics 22(1), 2020 DOI:10.1088/01430807/22/1/304

[2] Daniel Maystre, Maxwell: A new vision of the world, Comptes Rendus Physique 15 (5), 2014, 387 392, DOI:/10.1016/j.crhy.2014.02.004

[3] C. H. Chen et al., Effect of surrounding air region size on finite element modeling for permanent magnetic solenoids, journal of Applied Physics 109(7) DOI:10.1063/1.3540410

[4] Martín Martínez Villar, Finite element modelling of the magnetic field of guitar pickups with ANSYS DOI: $10.13140 /$ RG.2.1.3765.5920

[5] Dagoberto s. severo et al., Modeling magnetohydrodynamics of aluminum electrolysis cells with ANSYS and cfx, light metals 2005 edited by halvor kvande tms (the minerals, metals \& materials society), 2005

[6] A.A. Jadallah et al.Modeling and Simulation of a Photovoltaic Module in Different Operating Regimes, Acta Physica Polonica A, DOI: 10.12693/APhysPolA. 128.B-461

[7] B. Nagy, B. Thermal Calculation of Ground Contact Structures: New Methods Based on Parametrized Transient Finite Element Thermal Modeling, Acta Physica Polonica A, DOI: 10.12693/APhysPolA. 128.B-164

[8] Y. Özcanli et al. Comparison of Mechanical Properties and Artificial Neural Networks Modeling of PP/PET Blends, Acta Physica Polonica A, DOI:10.12693/APhysPolA.130.444

[9] A. Özdemir, State-Space Modeling of an EPW in Discrete Time and an Observer Design for State Variable Estimation, Acta Physica Polonica A, DOI:10.12693/APhysPolA.130.228

[10] I. Cayiroglu, Wing Aerodynamic Optimization by Using Genetic Algoritm and Ansys, Acta Physica Polonica A, DOI: 10.12693/APhysPolA.132.981

[11] A. Beycioğlua, Usability of Fuzzy Logic Modeling for Prediction of Fresh Properties of SelfCompacting Concrete, Acta Physica Polonica A, DOI: 10.12693/APhysPolA.132.1140 